This is an author produced version of a paper published in Environmental and Ecological Statistics. This paper has been peer-reviewed and is proof-corrected, but does not include the journal pagination.

Citation for the published paper:

Ramezani, H. \& Holm, S. (2009) Sample based estimation of landscape metrics; accuracy of line intersect sampling for estimating edge density and Shannon's diversity index. Environmental and Ecological Statistics.

Volume: 18 Number: 1, pp 109-130.

http://dx.doi.org/10.1007/s10651-009-0123-2

Access to the published version may require journal subscription.

Published with permission from: Springer

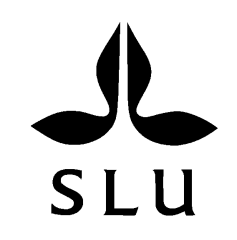

Epsilon Open Archive http://epsilon.slu.se 


\title{
Sample based estimation of landscape metrics; accuracy of line intersect sampling for estimating edge density and Shannon's diversity index
}

Authors: Habib Ramezani ${ }^{*}$, Sören Holm

${ }^{*}$ Ramezani. H (Corresponding author)

Department of Forest Resource Management, Swedish University of Agriculture

Sciences, SLU, SE-901 83 Umeå, Sweden

Phone: +46 9078681 51/ Fax: +46 90778116

E-mail: Habib.Ramezani@srh.slu.se, Ramezani.habib@gmail.com

\begin{abstract}
:
A recent trend is to estimate landscape metrics using sample data and costefficiency is one important reason for this development. In this study, line intersect sampling (LIS) was used as an alternative to wall-to-wall mapping for estimating Shannon’s diversity index and edge length and density. Monte Carlo simulation was applied to study the statistical performance of the estimators. All combinations of two sampling designs (random and systematic distribution of transects), four sample sizes, five transect configurations (straight line, L, Y, triangle, and quadrat), two transect orientations (fixed and random), and three configuration lengths were tested, each with a large number of simulations. Reference was 50 photos of size $1 \mathrm{~km}^{2}$, already manually delineated in vector format by photo interpreters using GIS environment. The performance was compared by root mean square error (RMSE) and bias. The best combination for all three metrics was found to be the systematic design and as response design the straight line configuration with random orientation of transects, with little difference between the fixed and random orientation of transects. The rate of decrease of RMSE for increasing sample size and line length was studied with a
\end{abstract}


mixed linear model. It was found that the RMSE decreased to a larger degree with the systematic design than the random one, especially with increasing sample size. Due to the nonlinearity in the definition of Shannon diversity estimator its estimator has a small and negative bias, decreasing with sample size and line length. Finally, a time study was conducted, measuring the time for registration of line intersections and their lengths on non-delineated aerial photos. The time study showed that long sampling lines were more cost-efficient than short ones for photo-interpretation.

Keywords: landscape pattern analysis, metric, Monte Carlo simulation, bias, root mean square error, wall-to-wall mapping, cost-efficiency

\section{Introduction}

Forest inventory is one of the most important applications of areal sampling (Williams 2001a, 2001b, Gregoire and Valentine 2008). Traditionally, the focus has been on information about timber production and plot sampling has been the dominant sampling method for this purpose. However, nowadays it is recognized that forests have many other values, e.g., as habitat for flora and fauna (FAO 2001, Corona et al. 2003) and a sampling method like line intersect sampling (LIS) (described in detail in § 2.2) is found to be efficient in many cases (e.g., Gregoire and Valentine 2008). Furthermore, with an increasing interest in nontimber forest values, forests cannot be treated independently from other land cover categories since the resource value of a forest often depends on the spatial context (Forman and Godron 1986). The demands for forest and landscape information have grown immensely during the last decades (Corona et al. 2003, Köhl 2003). Numerous large-scale environmental monitoring programs have been established and forest inventory methods are applied in such programs to collect more data on 
non-forest lands than before (NIJOS 2001, NILS 2003, Eiden et al. 2005). All this has resulted in a need for developing new areal sampling methods, or to adapt the existing methods to new situations.

The extension of the area of interest to all land categories has also been accompanied by new kinds of parameters of interest. To assess the ecological status of a landscape, ecologists have developed many different metrics in order to quantify landscape composition and configuration (McGarigal and Marks 1995, Gustafson 1998). Some aspects of landscape structure can be captured through Shannon diversity index and total edge length metrics (Kleinn C. 2000, Hernandez-Stefanoni 2006). The former one refers to both number of land cover types present and their proportions in landscape (Turner et al. 2001). The index value ranges between 0 and 1 . A high value indicates that land cover types have roughly equal proportion whereas a low value shows that landscape is dominated by one land cover type. An edge refers to the border between two different land cover types. It is a robust metric and is recommended as a fragmentation index ( $\mathrm{Li}$ et al. 1993, Saura and Martinez-Millan 2001). Depending on species under consideration the edge length in a landscape can have negative, positive and neutral impacts (Ries et al. 2004). There is a relationship between such landscape structures and ecological processes (Turner 1989) and the metrics are useful for understanding these relations (Leitão et al. 2006). They are increasingly used as indicators in monitoring programs, for comparisons on regional scale and changes over time (e.g., Hunsaker et al. 1994, NIJOS 2001, EPA 2003, Ji et al. 2006). Traditionally, complete land cover maps from remote sensing images are used for calculation of the metrics (e.g., Riitters et al. 1995, Herzog and Lausch 2001, Rocchini et al. 2006). The images are delineated into polygons of land cover types and the metrics are based on quantities like number of polygon, areas and edge 
lengths and on the spatial relationships between them. FRAGSTATS (McGarigal and Marks 1995) is a frequently used software for calculating landscape metrics from such data.

This method for calculating metrics is not without problems. Manual delineation of polygons on aerial photos is time-consuming (Corona et al. 2004) and is also apt to give systematic errors due to omission and commission errors (Carfagna and Gallego 1999). Classified maps from medium-resolution satellite images have low overall accuracy (Fang et al. 2006) while fine spatial resolution images entail huge amounts of data to be stored at high costs (Lu and Weng 2007). With automatized delineation less time is needed than manual but it can lead to increased costs due to the time required for correction. Furthermore adjacent land cover types are easily merged into one land cover type (Wulder et al. 2008). Finally, it is not certain that a delineation actually made is the proper one for all applications.

Considering these problems it is worthwhile asking whether or not a sampling approach could be a cost-efficient alternative. Several sampling and response designs are available, for photo interpretation as well as for field inventory. For instance, plot, line, and point sampling have been applied by Hunsaker et al. (1994), Corona et al. (2004) and Ramezani et al. (2009). Kleinn (2000) demonstrated that data from field-based forest inventories could be used to estimate some metrics to assess temporal landscape developments. Corona et al. (2004) found a sample survey to be a cost-efficient alternative to complete mapping. Further, probabilistic sampling allows to estimate precision and accuracy of estimates. It is well-known that in sample surveys the accuracy of observations on a restricted number of distinct plots tends to be higher than in total enumeration since data collection is more carefully made (Freese 1962, Raj 
1968). The sampling approach might also admit the use of data from ongoing surveys such as national forest inventories or to collect new data within these.

Line intersect sampling (LIS) has been used by several authors (e.g., Warren and Olsen 1964, Battles et al. 1996, Ringvall and Ståhl 1999a, Gregoire and Valentine 2003, Roth et al. 2003, Affleck et al. 2005) in various surveys. In some cases, LIS has shown to be an efficient method in field inventories and for remotely sensed data (e.g., Marshall et al. 2000, Esseen et al. 2006).

However, in general the papers referred to report specific applications of LIS for estimating conventional parameters like volume of coarse woody debris. The main objective of this paper is to study line intersect sampling for estimating landscape metrics. Different sampling designs and sample sizes and response designs as line configurations and their orientation and lengths have been tested on real landscape data. We assess the statistical performance of three different estimators of the three metrics Shannon diversity, edge length and edge density. In addition, the cost (time) of data collection is estimated in order to find costefficient alternatives.

\section{Method and material}

The study was accomplished as a sampling experiment, with line transects laid out over aerial photographs which had already been photo-interpreted in vector format. As we focused entirely on sampling errors, the manually photo-interpreted polygons (and the classes assigned to them) were assumed without errors. The complete maps were used as reference data for the sampling experiment. All combinations of systematic and random designs, four sample sizes, five transect configurations, fixed and random orientation of transects and three transect 
lengths were tested on two classification systems. The sampling properties of each combination of these sampling and response designs were estimated from a large number of Monte Carlo simulated samples for each delineated map. Both true and estimated values were calculated through a computer program, in FORTRAN, which was written specifically for this purpose.

\subsection{Study area}

The study was conducted in connection with the National Inventory of Landscape in Sweden (NILS; Allard et al. 2003), which is a major part of the national monitoring activities of the Swedish Environmental Protection Agency (EPA). In NILS, squares of 1 by $1 \mathrm{~km}$ are photo-interpreted. The focus of NILS is to get detailed land cover information and other data related to biodiversity. The digital photographs are in color infrared and have a ground resolution $0.4 \mathrm{~m}$ (scale 1:30,000). The interpretation is conducted through stereo observation, thereby also gaining information on the topography of the landscape. Within each squares, polygons are delineated and each described with 38 variables, using the interpretation program Summit Evolution from DAT/EM and ArcGIS from ESRI. From these variables, two hierarchical classification systems were designed: "level one” with seven classes and "level two" with twenty classes, to make land cover maps (Table 1). Fifty NILS's squares were used and true values of the metrics studied were obtained from the wall-to-wall mapping. 
Table1. Land cover categories identified with the two different classification systems. Level 1 contains seven classes and level 2 contains twenty classes.

\begin{tabular}{|c|c|}
\hline Land cover class level 1 & Land cover class level 2 \\
\hline \multirow[t]{6}{*}{ 1- Forest } & 1-1- Coniferous-Dense \\
\hline & 1-2- Coniferous-Sparse \\
\hline & 1-3- Deciduous-Dense \\
\hline & 1-4- Deciduous-Sparse \\
\hline & 1-5- Mixed-Forest- Dense \\
\hline & 1-6- Mixed-Forest- Sparse \\
\hline \multirow[t]{3}{*}{ 2- Urban } & 2-1- Housing-Areas \\
\hline & 2-2- Urban-Green-Areas \\
\hline & 2-3- Urban-Forest \\
\hline \multirow[t]{2}{*}{ 3- Cultivated fields } & 3-1-Crop-Fields \\
\hline & 3-2- Graze-Fields (grass land) \\
\hline \multirow[t]{3}{*}{ 4- Wetlands } & 4-1-Bog \\
\hline & 4-2- Fen \\
\hline & 4-3- Mixed-Wetland \\
\hline \multirow[t]{2}{*}{ 5- Water } & 5-1- Open-Water \\
\hline & 5-2- Water-Vegetation \\
\hline \multirow[t]{3}{*}{ 6- Pasture } & 6-1- Open- Pasture \\
\hline & 6-2- Pasture-Sparse-Trees \\
\hline & 6-3- Wooded-Pasture \\
\hline 7- Other land & 7- 1- Other land \\
\hline
\end{tabular}




\subsection{Line intersect sampling (LIS)}

LIS is a sampling method where population elements are included when a transect line crosses them (Thompson 2002). With this method a large (or long) object has a higher probability to be included in the sample than a small one. LIS is a wellknown and efficient method for sampling different kinds of features (Kaiser 1983, DeVries 1986, Skidmore and Turner 1992). It has been used in estimating total length of linear features (e.g., Matérn 1964, Corona et al. 2004), for measuring the area of two-dimensional objects (Battles et al. 1996), and also to estimate the total number of objects (Gregoire and Valentine 2008). In field-based inventories, imaginary lines between sample plots are line transects (e.g., Dahm 2001, NILS 2003). LIS can be implemented with different response designs as single straight lines transects or multiple-segmented transects such as the L-shape used in Canada, square transects such as in NILS (2003), or Y-shaped transects as used by U.S. Forest Service and the national forest inventory of Switzerland (Affleck et al. 2005). Irrespective of sampling design LIS can be implemented with random or fixed configuration orientation. Fixed means that the orientation of the transects is defined in advance, random that the orientations of the transects are independent and random. Yet, in the random case, inference may still condition on the orientation outcomes. The performance of transect orientation (random and fixed) with others combinations of line transects were studied by Hazard and Pickford (1986) in two simulated populations.

\subsection{Monte Carlo sampling simulation}

Monte Carlo sampling simulation has been used by several authors to study the statistical performance of estimators (Hazard and Pickford 1986, Ståhl 1998, Kleinn and Vilcko 2006, Ramezani et al. 2009). In this study, sampling simulation 
was used to estimate bias and RMSE of estimators of the Shannon diversity, edge length and edge density. Bias (or systematic error) is the difference between the expected value of the estimator and the true value. RMSE is the square root of the expected squared deviation between the estimator and the true value.

Sampling simulation was conducted for each combination two sampling designs (random and systematic), four sample sizes (16, 25, 49 and 100) and as response designs five line transect configurations (see Fig. 1), two transect orientations (fixed and random), three configuration lengths (37.5, 75 and $150 \mathrm{~m}$ ), two classification systems ( 7 and 20 classes). The direction of the systematic design and the fixed transect orientation is throughout north-south, the map orientation. In order to obtain roughly the same level of precision of the estimates of bias and RMSE for different sample sizes and transect lengths the number of replications were between 300 and 1000 depending on the total transect length in a sample. (In appendices 1 and 2 data on the precision of the estimated RMSE is given for a selection of combinations).

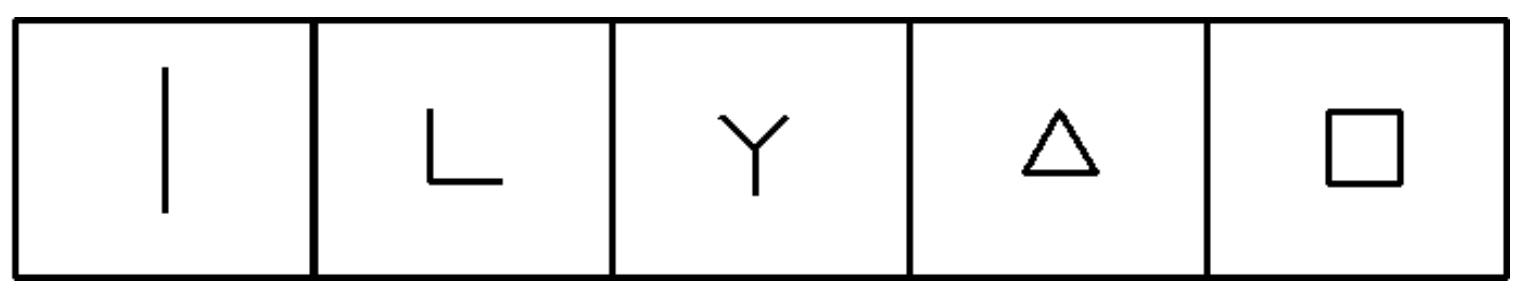

Figure 1. Illustration of the five different transect configurations applied in this study, line, L-shaped, Y-shaped, triangle and quadrat.

To avoid square edge effects the external peripheral method was applied (Gregoire and Valentine, 2008). The center of a line configuration was allowed to fall within a buffer zone of minimal width outside the map. Only intersections and lengths within the square were included. This method allows simple versions of 
the estimators. A drawback is that the sample size within the square is not fixed.

\subsection{Metrics estimators}

Metrics are defined through measurable characteristics of polygons (McGarigal and Marks 1995). In this study, area proportion and perimeter length of polygons were the basic properties for the metrics studied. The area $A_{j}$ of land cover type $\mathrm{j}$ was unbiasedly estimated by

$$
\hat{A}_{j}=\frac{A^{\prime}}{L} \cdot \sum_{i=1}^{n} l_{i j}
$$

where $l_{i j}$ is intersection length of the $j$ th land cover type with sampling line $i, L$ is the total length of all line transects, $A^{\prime}$ is the total area including the buffer, and $n$ is the sample size. The estimator was used for all five transect configurations.

\subsubsection{Shannon's diversity index}

Shannon diversity, $H$, is defined as

$$
H=-\frac{\sum_{j=1}^{s} p_{j} \cdot \ln \left(p_{j}\right)}{\ln (s)}
$$

where $s$ is the total number of land cover types considered (assumed known) and $p_{j}$ is the area proportion of the $j$ th land cover type. For $p_{j}=0, p_{j} \cdot \ln \left(p_{j}\right)$ is set

to zero. To estimate $H$, the area proportion was estimated by $\hat{p}_{j}=\hat{A}_{j} / \sum_{j=1}^{s} \hat{A}_{j}$ and inserted for $p_{j}$ in formula (2). Being a ratio estimator $\hat{p}_{j}$ is slightly biased. The estimator of $H$ can be shown to underestimate the true value. By a Taylor expansion, the absolute size of the bias can be shown to be approximately equal to 
$(t-1) /(2 \cdot n \cdot \ln (s))$, where $t$ is the number of land cover types actually present in the landscape.

\subsubsection{Edge length and edge density}

The estimators of total edge length and edge length for a single class were based on the method of Matérn (1964). The edge length can, given random sampling lines or edge directions, be estimated without bias by simply counting the number of intersections between a polygon border and line transects. According to Matérn (1964) and De Vries (1986), the total edge length estimator $\hat{T}$ ( $\left.\mathrm{m} \mathrm{ha}^{-1}\right)$, using multiple sampling lines of equal lengths, is given by

$$
\hat{T}=\frac{10000 \cdot \pi \cdot m}{2 \cdot n \cdot c}
$$

where $m$ is the total number of intersections, $c$ is the length of the sampling line per configuration (m), and $n$ is the sample size. For estimating edge density of a single land cover class $j$, the estimator used was $\hat{D}_{j}=\hat{T}_{j} / \hat{A}_{j}$, where $\hat{T}_{j}$ is the estimator (3) with $m=m_{j}$, the number of intersections with a class $j$ border. If $\hat{A}_{j}=0$ then $\hat{D}_{j}$ is undefined. It was also found during the simulations that $\hat{D}_{j}$ were very unstable if the number of intersections was low. Hence, for single class edge density only estimated values based on at least four intersections were accepted. This number was chosen arbitrarily but was found to be reasonable. A random distribution of straight lines on a land cover map with size $1 \mathrm{~km}^{2}$ is illustrated in Figure 2. 


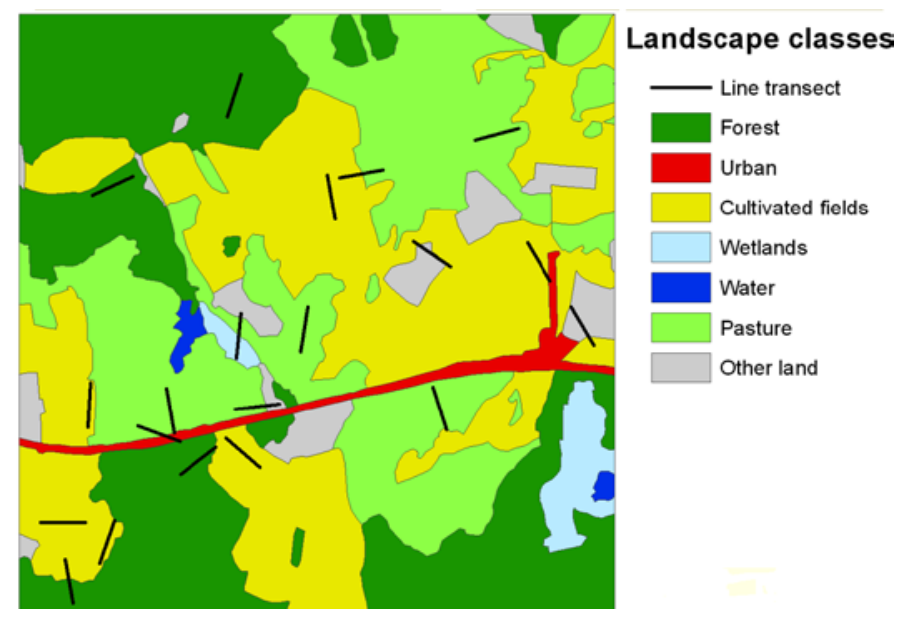

Figure 2. Illustration of random distribution of straight line transects on a land cover map $\left(1 \mathrm{~km}^{2}\right)$

\subsection{Quantification of errors}

The properties of the estimators were derived through a large number of independently simulated samples for each sample and response design and NILS square. For an estimator, $\hat{Y}$ say, the expected value was estimated by the mean over simulations

$$
\hat{E}(\hat{Y})=\frac{1}{M} \sum_{i=1}^{M} \hat{Y}_{i}
$$

where $\hat{Y}_{i}$ is the estimated value for the ith simulation and $M$ is the number of simulations. The estimated bias is $\hat{E}(\hat{Y})-Y$ where $Y$ is the true value. The root mean square error, RMSE, was estimated by

$$
R M S E=\sqrt{\sum_{i=1}^{M}\left(\hat{Y}_{i}-Y\right)^{2} / M}
$$

In case of an unbiased estimator, RMSE is the same as the estimated standard deviation of the estimator. Finally the mean value of the estimated bias and RMSE over the 50 squares was calculated. 
The results are reported as means over the 50 squares of the relative values for RMSE and bias. Furthermore, the efficiency of a sampling design should be evaluated in relation to costs and accuracy, as done for example by Ståhl (1998), Corona et al. (2004), Esseen et al. (2006), and Ramezani et al. (2009).

\subsection{Effect of sample size and line length on the RMSE}

The rate of decrease of RMSE with increasing sample size and/or line length was studied by the model

$$
z_{i j k}=\mu+c_{i}+\alpha \ln \left(n_{j}\right)+\beta \ln \left(l_{k}\right)+\lambda u_{j k}+e_{i j k}
$$

Here $z_{i j k}$ is the logarithm of the RMSE for square $i$, sample size $j$, line length $k$; $n_{j}$ and $l_{k}$ are sample size and length; $u_{j k}=\left(\ln \left(n_{j}\right)-m_{n}\right)\left(\ln \left(l_{k}\right)-m_{l}\right)$ where $m_{n}$ and $m_{l}$ are the means of $\ln \left(n_{j}\right)$ and $\ln \left(l_{k}\right)$ over the data set (here 3.6221 and 4.3175); $\mu, \alpha, \beta$ and $\lambda$ are fixed parameters; $c_{i}$ are random square effects with zero expectation and variance $\sigma_{c}^{2}$ and $e_{i j k}$ are random within square errors assumed to be uncorrelated to the $c_{i}$, with zero expectation and variance $\sigma_{e}^{2}$. A non-zero $\lambda$ shows interaction between size and length. The $u_{j k}$ are uncorrelated to $\ln \left(n_{j}\right)$ and $\ln \left(l_{k}\right)$, hence the estimates of $\alpha$ and $\beta$ will be the same whether the term $u_{j k}$ is included or not. The random variable $c_{i}$ expresses the general level of the RMSE of the square $i$. For random line location $\alpha$ should, in case of zero interaction, be at least approximately equal to -0.5 and for values of $\alpha$ smaller than -0.5 the decrease with sample size is faster than for random locations. For $\beta$, values larger than -0.5 are to be expected due to positive spatial autocorrelation. The larger the value of $\beta$ the less is the decrease of RMSE with line 
length. The parameter $\beta$ could be compared with the corresponding parameter of the so called Fairfield Smith's law for plot based sampling (Smith 1938). The variances of $c$ and $e$ tell us how much of the variation in RMSE is due to the variation between and within squares, respectively.

The model, if it is a good approximation, could be used to find an optimal sample size and line length by minimizing the expected RMSE (or its logarithm) for a given cost. The expected logarithmic RMSE is obtained by deleting the $c$ and $e$ terms in (6) and $C=n \cdot\left(C_{1}+C_{2} \cdot l^{\gamma}\right)$ is suggested as a fairly general cost function, where $C_{1}, C_{2}$ and $\gamma$ all are positive. Suppose first there is no interaction $(\lambda=0)$. Rearranging the cost formula in terms of $n$ and substitute this expression for $n$ into the expected logarithmic RMSE and upon taking the derivative we get $\frac{d R M S E}{d l}=G(l) \cdot\left[\frac{\beta C_{1}}{C_{2}}+l^{\gamma}(\beta-\gamma \alpha)\right]$, where $G(l)>0$ for all $l$. Since $\beta<0$ the derivative is negative if $\beta-\gamma \alpha \leq 0$ and $R M S E$ is then monotonically decreasing with line length. Otherwise the optimal line length is given by $l_{\text {opt }}=\left[\frac{C_{1}}{C_{2}} \cdot \frac{\beta}{(\alpha \gamma-\beta)}\right]^{1 / \gamma}$. If $\lambda \neq 0$ there is no explicit solution and we have to rely on numerical methods. Easiest is to substitute for $n$ as above and then calculate the RMSE for a number of lengths (using some search method).

\subsection{Time study}

The time needed for data acquisition was recorded for the line intersects sampling in a separate time study. An experienced (NILS) photo-interpreter counted the total number of intersections and determined the line lengths within different polygons for total edge length/density and Shannon diversity, respectively. In the case of forest edge density the interpreter measured the line lengths within the 
forest class polygons (seven class system) and counted the total number of intersections between forest class borders and transects. Only straight lines of lengths 37.5 and 150 meters were tested. The time study was performed on raw images (non-delineated aerial photos) and the photo-interpreter identified and counted borders among land cover types along transects without any previous polygon delineation. A moderate number of subjectively chosen NILS squares with good fragmentations were used. The interpreter had to find the lines manually in the image but the registrations were in principle made automatically. The time for pre-processing the images was not included. The average values are given in Table 2. The effect of the number of crossings had little effect on the time.

Table 2. Estimated time for two different lengths of sampling lines for edge length and Shannon diversity estimators. Straight line configuration. Level 1 classification system.

\begin{tabular}{|c|c|c|c|}
\hline \multirow[b]{2}{*}{$\begin{array}{l}\text { Sampling line length per } \\
\text { configuration (m) }\end{array}$} & \multicolumn{2}{|c|}{ Edge length estimators } & Shannon diversity estimator \\
\hline & $\begin{array}{r}\text { Average time }^{a} \\
\text { (minutes) }\end{array}$ & $\begin{array}{r}\text { Average time }^{b} \\
\text { (minutes) }\end{array}$ & $\begin{array}{c}\text { Average time }{ }^{c} \\
\text { (minutes) }\end{array}$ \\
\hline 37.5 & 0.12 & 0.15 & 0.19 \\
\hline 150 & 0.16 & 0.17 & 0.36 \\
\hline
\end{tabular}

${ }^{a}$ The estimated time to determine the line length within the forest class in minutes per line

${ }^{b}$ The estimated time for counting the number of intersections in minutes per line

${ }^{c}$ The estimated time to determine the line lengths within different classes in minutes per line

For the wall-to-wall mapping case, polygon delineation and classification of a NILS squares takes on average 3.5 hours (pre-processing not included) (Allard et al. 2007). To classify land along a line is not the same procedure as when polygon delineation is adopted. This does not affect the time study since the time for necessary detours from lines is included. Also, according to the photo interpreters 
involved, for the classification system used, the difficulties to classify are the same.

\section{Results}

\subsection{Shannon's diversity index}

The Relative RMSE of the Shannon diversity estimator $\hat{H}$, averaged over the 50 squares, for the level 1 classification system is shown in Figure 3.
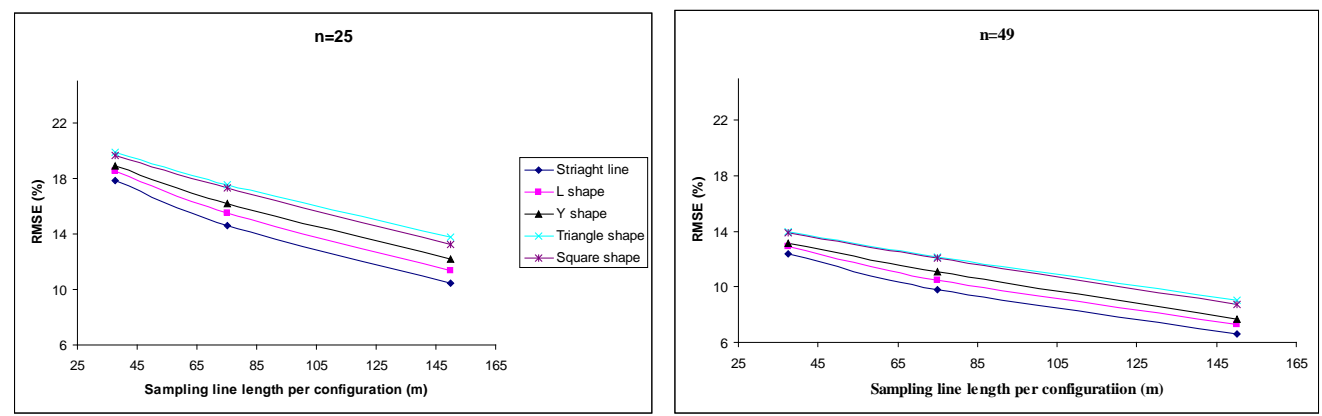

Figure 3. Relative RMSE of the Shannon diversity estimator for different sampling line lengths and configurations. Systematic sampling design, random orientation, level 1 classification system and two sample sizes (25 and 49)

For all three sampling line lengths, the systematic design resulted in a lower RMSE than did the random one. The true value of the Shannon diversity varied for the level 1 classification system between 0.024 and 0.787 over the 50 squares.

A comparison of the RMSE of Shannon diversity was made for the fixed and random orientations of the line transects. The random orientation of line transects resulted in a slightly lower RMSE. This was true for all five configurations, three different sampling line lengths and four sample sizes.

There was a negative and small bias for $\hat{H}$ though the area proportion of land cover type can be estimated almost without bias. (An example of true and estimated values of forest class area is provided in appendix 3). This is due to the nonlinearity in the definition of Shannon diversity estimator. In all combinations 
considered, the bias decreased with increasing number and length per configuration of sampling lines (Fig. 4).
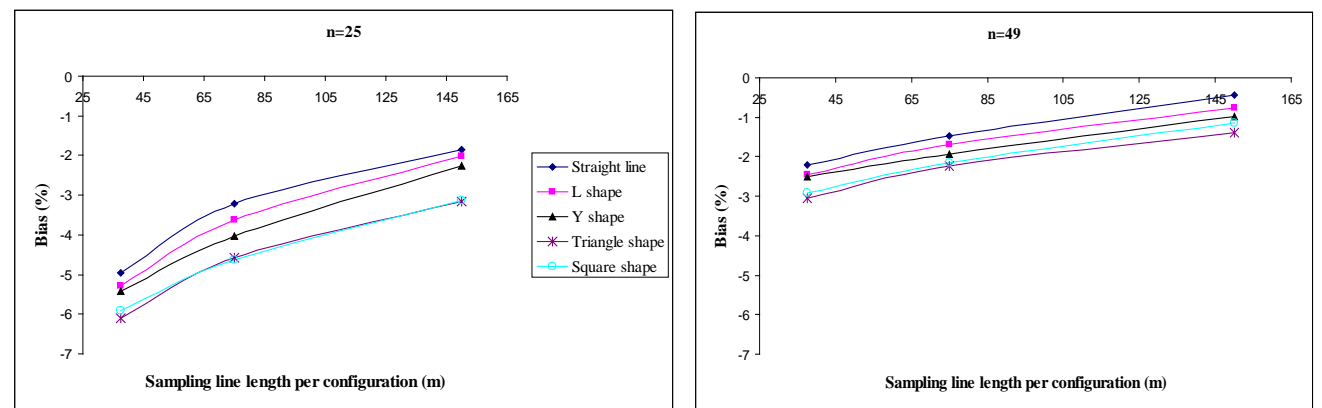

Figure 4. Relative bias of the Shannon diversity estimator for different sampling line lengths and configurations. Systematic design, random orientation of line transect, level 1 classification system and two sample sizes (25 and 49).

The general results were true in the level 2 classification system (with 20 classes) as well.

\subsection{Edge length and edge density}

The true values of the total edge density varied between 9.4 and $220.9\left(\mathrm{~m} \mathrm{ha}^{-1}\right)$ over the 50 squares. The relative RMSE of the total edge length estimator for the level 1 classification system is shown in Figure 5. Total edge density is, except for a scale factor, equivalent to total edge length.
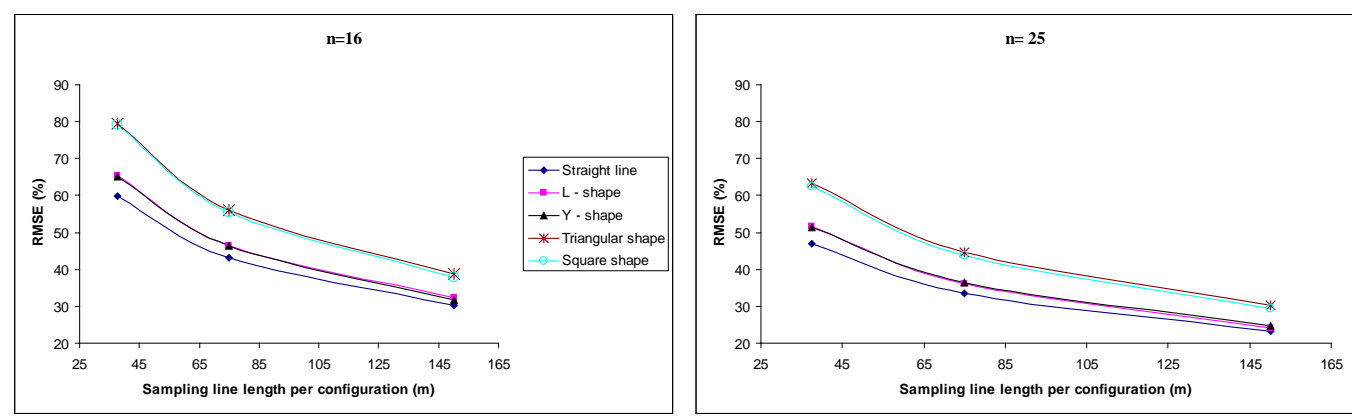

Figure 5. Relative RMSE of total edge length estimator for different sampling line lengths and configurations. Systematic design, random orientation, level 1 classification system and two sample sizes (16 and 25). 
A comparison of the relative RMSE of the total edge length estimator in two transect orientations showed that a random orientation of transects provided a lower RMSE in comparison to a fixed one (Fig. 6).

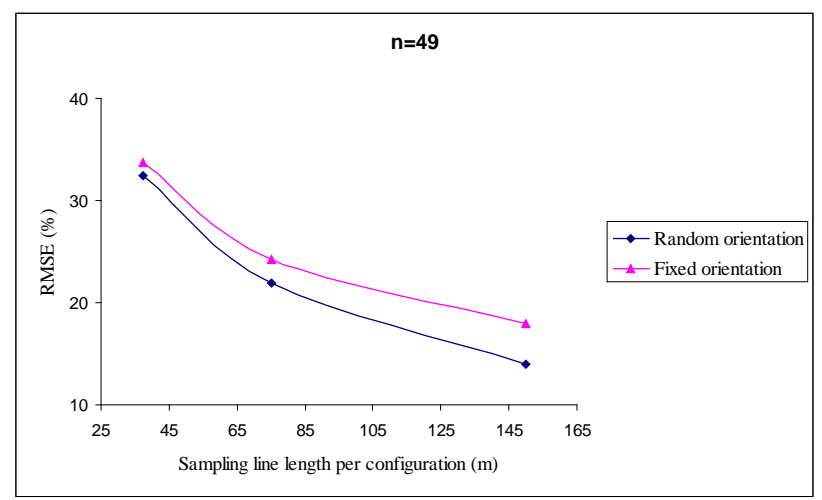

Figure 6. Relative RMSE of total edge length for the different orientations and sampling line lengths. Straight line and systematic design.

The edge length estimator was also studied for the single class of forest (level 1). The estimated relative RMSE for the forest class, using the systematic design and random orientation of transect lines, is shown in Figure 7. Five squares without any representation of the forest class were excluded. The RMSE of edge density for a single class estimator showed the same behavior as total edge length (density) on the landscape level. The true values of the edge density for the forest class varied between 9.5 and $505.7\left(\mathrm{~m} \mathrm{ha}^{-1}\right)$ over the 45 squares.
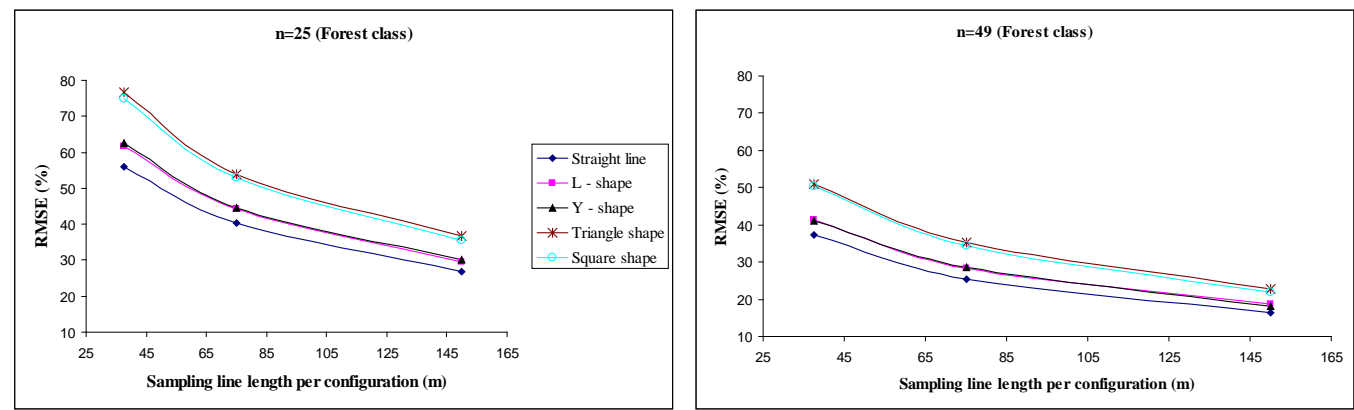

Figure 7. Relative RMSE of edge density for the forest class for different sample line lengths. Systematic design and random orientation and two sample sizes (25 and 49). 
In general, the results for all three metrics were as follows.

i) The systematic design was superior to the random one with respect to RMSE. This is in line with almost all areal sampling experiences. The reason is that the systematic design precludes observations at short distances, thus avoiding the negative effects of spatial auto-correlation.

ii) The straight line configuration gave the lowest RMSE, followed in pairs by the L- and Y-shape and finally by the triangular and quadrat configurations. The reason is the same as above. For a given configuration length, the straight line results in observations most far apart. For landscapes with gradients the L- and Yshapes could be more competitive. For field inventories the triangle and square shape have traveling distance advantages.

iii) The random orientation gave slightly less RMSE than the fixed one. The difference was small and might reflect some minor gradient in some or all of the landscapes studied.

\subsection{Effect of sample size and line length on the RMSE}

The parameters $\alpha, \beta$ and $\lambda$ in the model (6) were estimated for the systematic and random designs, the straight line and quadrat configurations, both with random orientation and for all three metrics. The estimated values of the parameters are shown in Table 3. 
Table 3. Estimated values of the parameters $\alpha$ (for sample size), $\beta$ (for line length), and $\lambda$ (for interaction) for systematic and random designs, for level 1 classification system. Standard errors are given within parentheses

\begin{tabular}{cccc}
\hline Population parameter & Parameter & Systematic design & Random design \\
\hline Straight line configuration & & & \\
\cline { 1 - 2 } Shannon's diversity & $\alpha$ & $-0.77(0.010)$ & $-0.55(0.004)$ \\
index & $\beta$ & $-0.41(0.012)$ & $-0.22(0.005)$ \\
& $\lambda$ & $-0.06(0.017)$ & $-0.00(0.007)$ \\
Total edge length & $\alpha$ & $-0.60(0.006)$ & $-0.50(0.002)$ \\
& $\beta$ & $-0.57(0.007)$ & $-0.41(0.003)$ \\
Edge density for the & $\alpha$ & $-0.07(0.010)$ & $-0.00(0.004)$ \\
forest class & $\beta$ & $-0.65(0.007)$ & $-0.55(0.003)$ \\
Quadrat configuration & $\lambda$ & $-0.56(0.009)$ & $-0.40(0.004)$ \\
Shannon's diversity & $\alpha$ & $-0.05(0.012)$ & $-0.00(0.006)$ \\
index & $\beta$ & & \\
& $\lambda$ & $-0.77(0.012)$ & $-0.54(0.004)$ \\
Total edge length & $\beta$ & $-0.37(0.015)$ & $-0.18(0.005)$ \\
& $\alpha$ & $-0.59(0.008)$ & $-0.50(0.002)$ \\
Edge density for the & $\alpha$ & $-0.60(0.009)$ & $-0.46(0.003)$ \\
forest class & $\beta$ & $-0.10(0.013)$ & $-0.00(0.004)$ \\
& $\lambda$ & $-0.65(0.009)$ & $-0.53(0.003)$ \\
& & $-0.60(0.011)$ & $-0.43(0.004)$ \\
& & $-0.11(0.015)$ & $-0.00(0.006)$ \\
\hline
\end{tabular}

The values for the random design are as expected, with no interaction. For the systematic design a certain interaction was found and its sign shows that an increase in configuration length is relatively more favorable for large sample sizes than for small. For example, for the Shannon index the marginal $\beta$ equals -0.37 for the sample size 16 and -0.45 for the sample size 100 . The values for the two configurations are very close, except for the interaction. Especially for the Shannon diversity the decrease in RMSE with sample size is faster for the systematic design than for the random one.

The fit was good, with adjusted R-square over $91 \%$ in all 12 cases. The variance $\left(\sigma_{c}^{2}\right)$ between squares were considerably higher than within $\left(\sigma_{e}^{2}\right)$, in 10 
of the cases it contributed to over $90 \%$ of the total variance.

\subsection{Time study}

The relationships between RMSE of estimators and time (cost) for estimating total edge length (density), edge density for the forest class and Shannon diversity are shown in Figure 8. In the case of total edge length, with a given sampling budget, the magnitude of RMSE decreased with the length of the sampling lines. The same was true for the edge density estimator for the forest class. In the Shannon diversity case, however, with a given cost, there was no difference between long and short lines. 

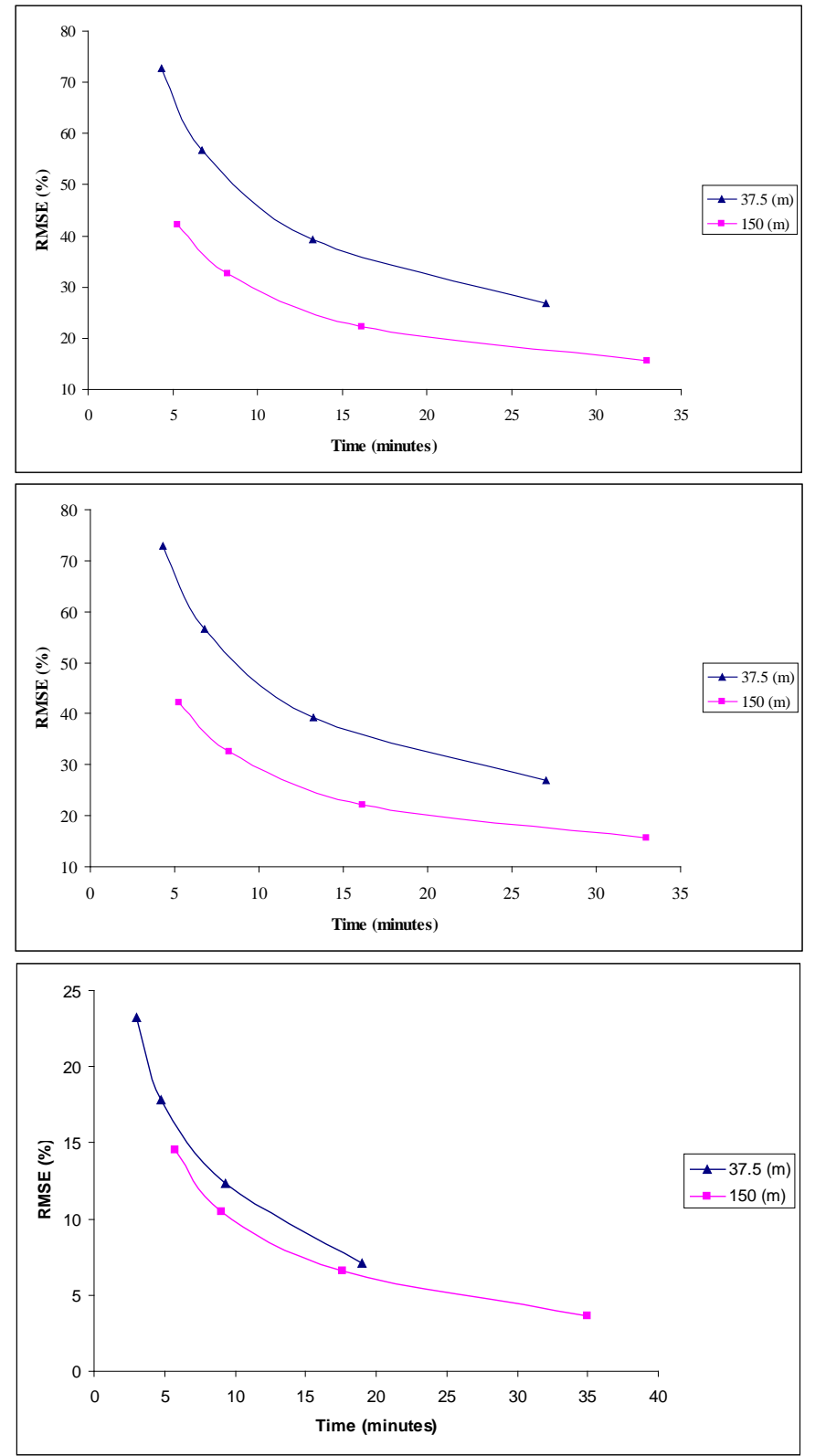

Figure 8. Relationships between time (cost) and the RMSE of the total edge length /density (top), edge density for the forest class (middle) and Shannon diversity (bottom) estimators for different sampling line lengths, according to Table 2. Random design, straight line.

About the same results were obtained by the model (6) and the cost function suggested. The results are not general and should be seen as an example. A value of $C_{1}$ corresponding to 5 seconds seemed realistic from the time study. The remaining parameters of the cost function was equated to the observed average times and the values obtained were $0.5,2.0$ and 0.32 for $C_{2}$ and $0.7,0.2$ and 0.5 for $\gamma$ for the Shannon diversity, total edge length and forest class edge density 
respectively. Due to the interaction the optimal transect length for the systematic design depends on the total resource $C$ and more resources leads, due to the sign of $\lambda$, to longer transects as long as they are within the allowable range. For edge length and edge density the maximum length of 150 meters was optimal within the range and markedly superior to short lengths, both for systematic and random design. For the Shannon diversity and systematic design the optimal length, given a resource of 10 minutes per square, was about 70 meters. For the random design it was 140 meters. However, for the Shannon diversity the difference in RMSE, for a given cost, differed from the minimum RMSE by only a few percent within the allowable range. Hence, the RMSE could, for a given cost, be considered as almost independent of line length. It should be noted that for field inventories the parameters of the cost function will be quite different, with $\gamma \geq 1$ for example, favoring short line lengths.

\section{Discussion}

This study indicates the potential of using sample-based assessment of landscape

metrics by line intersect sampling (LIS) as an alternative to wall-to-wall mapping for estimating the basic metrics Shannon diversity and edge length/density. The sample design can be used both in remote sensing imagery and in the field.

Total length of linear features in landscape can be estimated without bias using LIS if sample lines and/or population of interest (objects) are randomly oriented (DeVries 1979, Kaiser 1983). Corona et al. (2004) found, on aerial photos, that LIS can result in an accurate estimate of total edge length. They found LIS to be more efficient than the traditional method of polygon delineation and found longer sampling lines to be better than short ones, similarly as in the present study. They did not find any significant difference between systematic and 
random designs. The authors did not test other transect configurations than the straight line. LIS was also used by Hansen (1985), Dahm (2001) and Matérn (1964) for estimating total length of linear landscape features.

LIS has been used in sample surveys for different purposes. For instance, it was applied by Hazard and Pickford (1986) in estimating forest residue. They compared three different configurations straight line, L, and Y shapes in two different simulated spatial patterns of forest residue. They concluded that, for a simulated population, $\mathrm{L}$ and $\mathrm{Y}$ shapes were more efficient than the straight line configuration. The authors also found that systematic sampling was more efficient than the random design. Ringvall and Ståhl (1999b) and Fjellstad and Dramstad (1999) used LIS to assess parameter values for sparse populations and to estimate changes in areas and transitions between land cover types, respectively.

Statistical performance of estimators of some landscape metrics were discussed by Hunsaker et al. (1994) using plot sampling method on satellite-based land cover map. The shape index in term of fractal geometry and the dominance (equivalent to the Shannon diversity) metric had bias whereas the contagion metric was unbiasedly estimated.

Point sampling (dot grid) was applied by Ramezani et al. (2009) for estimating Shannon diversity and edge length metrics, where the area of a buffer generated around polygon borders was used for estimating edge length. The authors concluded that the method appears to be an efficient alternative to wall-to-wall approach.

For the study, 50 randomly selected Swedish landscape maps from the NILS monitoring program were used. There were several reasons for choosing real landscapes instead of simulated. The methods studied are intended for application on real landscapes why the results are of direct use. By simulation it is not likely 
that the large variation in landscape patterns that exist in reality is created. Also the time study required real photos. A drawback is that we cannot deliberately create patterns to stress certain properties of the methods.

All results reported are obtained for the estimator based on an assumption of completely random direction of sampling lines and/or population edges. Two other estimators were also tested, the basic Horvitz-Thompson estimator, conditional on the intersection angle (Thompson 2002) and an estimator assuming a uniform angle distribution within which of the three thirty-degree sectors 0-30, $30-60$ or $60-90$ degrees the intersection was observed. The estimator is obtained by changing the factor $\pi / 2$ in the basic estimator used to $\pi(2+\sqrt{3) / 3}$, $\pi(1+\sqrt{3}) / 6$ or $\pi / 3$ depending on sector. The factors are the inverses of expected $\sin (v)$ within the sector. The RMSE was found to be considerably larger for the two alternative estimators and all cases studied.

The results of a statistical model for RMSE demonstrated that the effect of line length is larger for both designs for the two edge parameters than for the Shannon diversity. For the systematic design and the edge parameters the result even indicates negative auto-correlation of edge intersections along sampling lines. This could be explained by some regularity in the landscapes and that few very small polygons are present, implying that clusters of intersections are rare. The estimator of the Shannon diversity is to a high degree based on area estimation and its RMSE is not much affected by such spatial regularity. The estimates of the two variance components of the model revealed, not surprisingly, that the total variation of (logarithmic) RMSE, for given line length and sample size, was mostly due to a variation of RMSE among squares, not within. By estimating their fixed counterparts it is possible to find what landscape features determine the 
magnitude of the RMSE. For Shannon diversity it was found that the larger the actual number of cover classes present in the square, the larger the RMSE; however considerable variation still exists. For total edge length almost all of the variation in logarithmic RMSE over squares was explained by the true (logarithmic) total edge length, and the larger the edge length, the larger the RMSE. For edge density of the forest class, the true edge density explained some of the variation, but so did also the total forest area. The RMSE increased with true density and decreased with increasing area, the latter reflecting the effect on the density estimator of the area estimator part, for which the relative RMSE decreases with true area.

The time study revealed no large differences in interpreting times between short and long line lengths, which favors the latter alternative with respect to costefficiency. One reason is naturally that the scale on the interpreter's screen could be adjusted for the lengths. Another reason is that a non-negligible part of the time was spent to move the cursor between the different lines. The number of intersections had very little effect on the time except if they were very few. The average time needed to determine the lengths within the forest class was shorter than to determine the number of intersections. This is explained by lines being easily recognized to be entirely within or outside the forest class, thus many intersections were between other classes, and that the lengths were quickly measured by a computer application. Another application, not used but possible to implement, consists in finding the lines automatically and this would likely change the time relation between short and long lines. The results of the time study should thus be considered with caution due to available computer facilities and also to the complexity of the landscape (i.e. for a more detailed classification system than used here). The main result of the time study is, in the present case, 
nevertheless, that long lines are cost-efficient, perhaps even longer than those tested here. To judge where LIS is cost-efficient compared to wall-to-wall mapping we have to know the desired accuracy for different parameters, for example total edge length. According to Figure 8 it is possible to get an expected relative RMSE of about 15 percent within half an hour, whereas complete wall-towall mapping of the same area takes about 3.5 hours. In the Shannon diversity case, it takes half an hour for achieving a RMSE of about 1 in absolute term, with line $150 \mathrm{~m}$.

In sample based assessment of landscape metrics, the efficiency of a sampling and response design depends on the selected metric. For instance, point sampling may be a more efficient method than LIS for Shannon diversity through area estimation. In contrast, LIS might be preferable to point sampling for estimating total edge length. Hence, for estimating a more complex metric like edge density for a certain class (e.g., forest), a LIS design could be used for the edge length and a point sampling design for the area. This suggests more complex designs, for example, using a LIS with central points in the lines, supplemented with extra points for area estimation and the lines for edge length estimation. Another area for further investigation is two-stage designs for estimating metrics for large landscapes, where squares can be used as first stage sampling units.

\section{Acknowledgment}

We are grateful to Prof. Göran Ståhl for constructive comments. The authors are also grateful to Mats Högström for help with ArcGIS and Björn Nilsson for help with the time study; all three at SLU in Umeå, Sweden. We would also like to thank three anonymous reviewers for their helpful comments on manuscript. 


\section{Reference}

1) Affleck D. L. R., Gregoire T. G. and Valentine H. T. (2005). Design unbiased estimation in line intersect sampling using segmented transects. Environmental and Ecological Statistics 12:139-154.

2) Allard A., Esseen P-A. , Holm S., Högström M., Marklund L., Nilsson B., et al. (2007). Fångst av vegetationsdata och Natura 2000-habitat i fjällen genom flygbildstolkning i IRF med punktgittermetodik (Catch of vegetation data and the Natura 2000 habitats in the mountains by Localization interpretation of the IRF with point lattice method). Swedish University of Agricultural Sciences, Department of Forest Resource Management, Work Report 172 (in Swedish).

3) Allard A., Nilsson B., Pramborg K., Ståhl G. and Sundquist S. (2003). Manual for Aerial Photo Interpretation in the National Inventory of Landscapes in Sweden NILS. Swedish University for Agricultural Sciences, SLU, Umeå, Sweden.

4) Battles J., Dushoff G. and Fahey J. (1996). Line intersects sampling of forest canopy gaps. Forest Science 42:131-138.

5) Carfagna E. and Gallego F. (1999). Thematic maps and statistics. in Land cover and land use information systems for European Union policy needs. Office for Official publications of the European Communities, Luxembourg.pp.219-228.

6) Corona P., Chirici G. and Travaglini D. (2004). Forest ecotone survey by line intersect sampling. Canadian Journal of Forest Research-Revue Canadienne De Recherche Forestiere 34:1776-1783.

7) Corona P., Köhl M. and Marchetti M. (2003). Advance in forest inventory for sustainable forest management and biodiversity monitoring. Kluwer academic, Netherlands.

8) Dahm S. (2001). Investigation of forest edge lengths based on data of the first Federal Forest Inventory. Allgemeine Forst Und Jagdzeitung 172:8186.

9) DeVries P.G. (1979). Line intersect sampling: statistical theory, applications, and suggestions for extended use in ecological inventory. in Cormack R. M., Patil G. P., and Robson D. S., editors. Sampling biological population. International Co-operative publishing house, Fairland, Maryland, pp. 1-70.

10) DeVries P.G. (1986). Sampling theory for forest inventory. Springer Verlag, Berlin, Germany.

11) Eiden G., Jadues P. and Theis R. (2005). Linear landscape features in the European Union. Developing indicators related to linear landscape features based on LUCAS transect data an EU publication report EUR 21669 "Trends of some agri-environmental indicators in the European Commission".

12) EPA. (2003). Environmental Monitoring Program. Swedish Environmental Protection Agency.

13) Esseen P. A., Jansson K. U. and Nilsson M. (2006). Forest edge quantification by line intersect sampling in aerial photographs. Forest Ecology and Management 230:32-42.

14) Fang S. F., Gertner G., Wang G. X. and Anderson A. (2006). The impact of misclassification in land use maps in the prediction of landscape dynamics. Landscape Ecology 21:233-242. 
15) FAO. (2001). State of forests 2001. FAO Repot, Rome, Italy.

16) Fjellstad W. J. and Dramstad W. E. (1999). Patterns of change in two contrasting Norwegian agricultural landscapes. Landscape and Urban Planning 45:177-191.

17) Forman R. T. T. and Godron M. (1986). Landscape ecology John Wiley \& Sons, New York.

18) Freese F. (1962). Elementary forest sampling. USDA Forest service, Washington D.C.

19) Gregoire T. G. and Valentine H. T. (2003). Line intersect sampling: Ellshaped transects and multiple intersections. Environmental and Ecological Statistics 10:263-279.

20) Gregoire T. G. and Valentine Harry T. (2008). Sampling Strategies for Natural Resources and the Environment Chapman \& Hall/CRC, Boca Raton, Fla. London.

21) Gustafson J. E. (1998). Quantifying landscape spatial pattern: What is the state of the art? Ecosystems 1:143-156.

22) Hansen H. (1985). Line intersect sampling of wooded strips . Forest Science 31:282-288.

23) Hazard J. and Pickford S. (1986). Simulation studies on Line intersect sampling of forest residue, part II. Forest Science 32:447-470.

24) Hernandez-Stefanoni J. L. (2006). The role of landscape patterns of habitat types on plant species diversity of a tropical forest in Mexico. Biodiversity and Conservation 15:1441-1457.

25) Herzog F. and Lausch A. (2001). Supplementing land-use statistics with landscape metrics: Some methodological considerations. Environmental Monitoring and Assessment 72:37-50.

26) Hunsaker C. T., O'Neill R. V., Jackson B. L., Timmins S. P., Levine D. A. and Norton D. J. (1994). Sampling to characterize landscape pattern. Landscape Ecology 9:207-226.

27) Ji W., Ma J., Twibell R. W. and Underhill K. (2006). Characterizing urban sprawl using multi-stage remote sensing images and landscape metrics. Computers Environment and Urban Systems 30:861-879.

28) Kaiser L. (1983). Unbiased estimation in line-intercept sampling. Biometrics 39:965-975.

29) Kleinn C. (2000). On large area inventory and assessment of trees outside forests. Unasylva 200:3-10.

30) Kleinn C. (2000). Estimating metrics of forest spatial pattern from large area forest inventory cluster samples. Forest Science 46:548-557.

31) Kleinn C. and Vilcko F. (2006). A new empirical approach for estimation in k-tree sampling. Forest Ecology and Management 237:522-533.

32) Köhl M. (2003). New approaches for multi-resource forest inventories. in Corona P., Köhl M., and Marchetti M., editors. Advance in forest inventory for sustainable forest management and biodiversity monitoring. .Kluwer Academic, Netherlands.

33) Leitão A. , Miller J. and Aher J. (2006) Measuring landscapes: a planner's handbook. Island Press, Washington, DC.

34) Li H. B., Franklin J. F., Swanson F. J. and Spies T. A. (1993). Developing alternative forest cutting patterns - a simulation approach. Landscape Ecology 8:63-75. 
35) Lu D. and Weng Q. (2007). A survey of image classification methods and techniques for improving classification performance. International Journal of Remote Sensing 28:823-870.

36) Marshall P.L., Davis G. and LeMay V.M. (2000). Using line intersect sampling for coarse woody debris. Technical Report TR-003, Research Section, Vancouver Forest Region, British Columbia Ministry of Forests.

37) Matérn B. (1964). A method of estimating the total length of roads by means of line survey. Studia forestalia Suecica 18:68-70.

38) McGarigal K. and Marks E. J. (1995). FRAGSTATS: Spatial pattern analysis program for quantifying landscape pattern. General Technical Report 351. U.S. Department of Agriculture, Forest Service, Pacific Northwest Research Station.

39) NIJOS. (2001). Norwegian 3Q Monitoring Program. Norwegian institute of land inventory.

40) NILS. (2003). National Inventory of Landscapes in Sweden. SLU, department of forest resource management. Umeå.

41) Raj D. (1968). Sampling theory. McGraw-Hill, New York.

42) Ramezani H., Holm S., Allard A. and Ståhl G. (2009). Monitoring landscape metrics by point sampling: accuracy in estimating Shannon's diversity and edge density. Environmental Monitoring and Assessment (in press).

43) Ries L., Fletcher R. J., Battin J. and Sisk T. D. (2004). Ecological responses to habitat edges: Mechanisms, models, and variability explained. Annual Review of Ecology Evolution and Systematics 35:491522.

44) Riitters K. H., O'Neill R. V., Hunsaker C. T., Wickham J. D., Yankee D. H., Timmins S. P., et al. (1995). A factor-analysis of landscape pattern and structure metrics. Landscape Ecology 10:23-39.

45) Ringvall A. and Ståhl G. (1999a). Field aspects of line intersect sampling for assessing coarse woody debris. Forest Ecology and Management 119:163-170.

46) Ringvall A. and Ståhl G. (1999b). On the field performance of transect relascope sampling for assessing downed coarse woody debris.

Scandinavian Journal of Forest Research 14:552-557.

47) Rocchini D., Perry G. L. W., Salerno M., Maccherini S. and Chiarucci A. (2006). Landscape change and the dynamics of open formations in a natural reserve. Landscape and Urban Planning 77:167-177.

48) Roth A., Kennel E., Knoke T. and Matthes U. (2003). Line intersect sampling: An efficient method for sampling of coarse woody debris? Forstwissenschaftliches Centralblatt 122:318-336.

49) Saura S. and Martinez-Millan J. (2001). Sensitivity of landscape pattern metrics to map spatial extent. Photogrammetric Engineering and Remote Sensing 67:1027-1036.

50) Skidmore A. K. and Turner B. J. (1992). Map Accuracy Assessment Using Line Intersect Sampling. Photogrammetric Engineering and Remote Sensing 58:1453-1457.

51) Smith H. F. (1938). An empirical law describing heterogeneity in the yields of agricultural crops. Journal of Agricultural Science 28.

52) Ståhl G. (1998). Transect relascope sampling - A method for the quantification of coarse woody debris. Forest Science 44:58-63.

53) Thompson S. K. (2002). Sampling, 2nd. edition. Wiley, New York. 
54) Turner M. G. (1989). Landscape ecology: The effect of pattern on process. Annual Review of Ecology and Systematics 20:171-197.

55) Turner M. G., Gardner R. H. and O'Neill R. V. (2001). Landscape ecology in theory and practice : pattern and process. Springer, New York.

56) Warren W.G. and Olsen P.F. (1964). A line intesect technique for assessing logging waste. Forest Science 10:267-276.

57) Williams M. S. (2001a). New approach to areal sampling in ecological surveys. Forest Ecology and Management 154:11-22.

58) Williams M. S. (2001b). Nonuniform random sampling: an alternative method of variance reduction for forest surveys. Canadian Journal of Forest Research-Revue Canadienne De Recherche Forestiere 31:20802088.

59) Wulder M. A., White J. C., Hay G. J. and Castilla G. (2008). Towards automated segmentation of forest inventory polygons on high spatial resolution satellite imagery. Forestry Chronicle 84:221-230. 
Appendix 1. Estimated relative range and mean of SE of RMSE for Shannon diversity over the 50 squares for selection of sampling and response designs, obtained by 10 replications of each case and square.

\begin{tabular}{|c|c|c|c|c|c|c|}
\hline \multicolumn{4}{|c|}{ Parameter set } & \multirow[b]{2}{*}{$\begin{array}{l}\text { Sample } \\
\text { replication }\end{array}$} & \multirow[b]{2}{*}{ Range } & \multirow[b]{2}{*}{ Mean } \\
\hline $\begin{array}{l}\text { Sampling } \\
\text { Design }^{\text {a }}\end{array}$ & $\begin{array}{l}\text { Sample } \\
\text { size }\end{array}$ & Conf. $^{\text {b }}$ & $\begin{array}{c}\text { Length }^{\mathrm{c}} \\
\text { (m) }\end{array}$ & & & \\
\hline 1 & 16 & 1 & 1 & 1000 & $0.9-4.5$ & 2.3 \\
\hline 1 & 16 & 3 & 1 & 1000 & $0.7-4.0$ & 2.1 \\
\hline 1 & 16 & 5 & 1 & 1000 & $0.6-3.2$ & 2.1 \\
\hline 1 & 16 & 1 & 3 & 600 & $1.3-4.5$ & 2.7 \\
\hline 1 & 16 & 3 & 3 & 600 & $1.5-5.1$ & 3.0 \\
\hline 1 & 16 & 5 & 3 & 600 & $1.2-4.8$ & 2.8 \\
\hline 1 & 25 & 1 & 2 & 800 & $1.1-3.7$ & 2.3 \\
\hline 1 & 49 & 1 & 2 & 600 & $1.1-3.7$ & 1.1 \\
\hline 1 & 100 & 1 & 1 & 600 & $1.2-3.9$ & 2.4 \\
\hline 1 & 100 & 1 & 3 & 300 & 2.3-6.1 & 3.8 \\
\hline 2 & 16 & 1 & 1 & 1000 & $1-4.4 .0$ & 2.3 \\
\hline 2 & 16 & 3 & 1 & 1000 & $1.4-4.6$ & 2.3 \\
\hline 2 & 16 & 5 & 1 & 1000 & $1.4-4.9$ & 2.3 \\
\hline 2 & 25 & 1 & 2 & 800 & $1.7-4.5$ & 2.7 \\
\hline 2 & 49 & 1 & 2 & 600 & $1.6-5.3$ & 3.0 \\
\hline 2 & 100 & 1 & 1 & 600 & $1.5-4.7$ & 3.0 \\
\hline 2 & 100 & 1 & 3 & 300 & $1.6-6.2$ & 4.1 \\
\hline
\end{tabular}

${ }^{\mathrm{a}}$ Sampling design; Systematic $=1$, Random $=2$

${ }^{\mathrm{b}}$ Configuration; Straight line $=1, \mathrm{Y}=3$, Quadrat $=5$

${ }^{\mathrm{c}}$ Configuration length; 37.5 $=1,75=2,150=3$ 
Appendix 2. Estimated relative range and mean of SE of RMSE for total edge length and edge density of forest class over the 50 squares for selection of sampling and response designs, obtained by 10 replications of each case and square

\begin{tabular}{|c|c|c|c|c|c|c|}
\hline \multicolumn{4}{|c|}{ Parameter set } & \multirow{2}{*}{$\begin{array}{l}\text { Sample } \\
\text { replication }\end{array}$} & \multirow[b]{2}{*}{ Range } & \multirow[b]{2}{*}{ Mean } \\
\hline $\begin{array}{l}\text { Sampling } \\
\text { Design }^{\mathrm{a}}\end{array}$ & $\begin{array}{c}\text { Sample } \\
\text { size }\end{array}$ & Conf. $^{\mathrm{b}}$ & $\begin{array}{c}\text { Length }^{c} \\
\text { (m) }\end{array}$ & & & \\
\hline \multicolumn{7}{|c|}{ Total edge length } \\
\hline 1 & 16 & 1 & 1 & 1000 & $1.0-4.9$ & 2.4 \\
\hline 1 & 16 & 3 & 1 & 1000 & $1.4-3.8$ & 2.4 \\
\hline 1 & 16 & 5 & 1 & 1000 & $1.1-5.1$ & 2.2 \\
\hline 1 & 16 & 1 & 3 & 600 & $1.1-5.9$ & 2.8 \\
\hline 1 & 16 & 3 & 3 & 600 & $1.5-5.7$ & 2.8 \\
\hline 1 & 16 & 5 & 3 & 600 & $1.4-4.4$ & 2.7 \\
\hline 1 & 25 & 1 & 2 & 800 & $1.2-3.7$ & 2.4 \\
\hline 1 & 49 & 1 & 2 & 600 & $1.2-5.6$ & 2.8 \\
\hline 1 & 100 & 1 & 1 & 600 & $0.9-5.1$ & 2.3 \\
\hline 1 & 100 & 1 & 3 & 300 & $2.3-6.3$ & 3.9 \\
\hline 2 & 16 & 1 & 1 & 1000 & $1.1-5.3$ & 2.5 \\
\hline 2 & 16 & 3 & 1 & 1000 & $1.7-4.6$ & 2.6 \\
\hline 2 & 16 & 5 & 1 & 1000 & $1.1-6.8$ & 2.5 \\
\hline 2 & 25 & 1 & 2 & 800 & $1.2-4.2$ & 2.5 \\
\hline 2 & 49 & 1 & 2 & 600 & $1.6-4.7$ & 2.7 \\
\hline 2 & 100 & 1 & 1 & 600 & $1.3-4.1$ & 2.5 \\
\hline 2 & 100 & 1 & 3 & 300 & $1.6-6.5$ & 4.0 \\
\hline \multicolumn{7}{|c|}{ Edge density of forest class } \\
\hline 1 & 16 & 1 & 1 & 1000 & $1.7-12.4$ & 3.5 \\
\hline 1 & 16 & 3 & 1 & 1000 & $1.3-13.1$ & 3.5 \\
\hline 1 & 16 & 5 & 1 & 1000 & $1.4-15.6$ & 3.4 \\
\hline 1 & 16 & 1 & 3 & 600 & $1.1-13.4$ & 3.3 \\
\hline 1 & 16 & 3 & 3 & 600 & $1.6-7.4$ & 3.2 \\
\hline 1 & 16 & 5 & 3 & 600 & $1.5-7.2$ & 3.3 \\
\hline 1 & 25 & 1 & 2 & 800 & $1.2-4.3$ & 2.6 \\
\hline 1 & 49 & 1 & 2 & 600 & $1.6-6.6$ & 3.1 \\
\hline 1 & 100 & 1 & 1 & 600 & $0.6-5.0$ & 2.2 \\
\hline 1 & 100 & 1 & 3 & 300 & $2.6-6.2$ & 4.1 \\
\hline 2 & 16 & 1 & 1 & 1000 & $1.6-17.3$ & 4.5 \\
\hline 2 & 16 & 3 & 1 & 1000 & $2.1-20.4$ & 4.6 \\
\hline 2 & 16 & 5 & 1 & 1000 & $1.1-29.5$ & 4.8 \\
\hline 2 & 25 & 1 & 2 & 800 & $1.4-10.0$ & 3.4 \\
\hline 2 & 49 & 1 & 2 & 600 & $1.8-7.0$ & 3.1 \\
\hline 2 & 100 & 1 & 1 & 600 & $0.6-5.2$ & 2.6 \\
\hline 2 & 100 & 1 & 3 & 300 & $2.1-7.1$ & 4.2 \\
\hline
\end{tabular}

${ }^{\mathrm{a}}$ Sampling design; Systematic $=1$, Random $=2$

${ }^{\mathrm{b}}$ Configuration; Straight line $=1, \mathrm{Y}=3$, Quadrat $=5$

${ }^{\mathrm{c}}$ Configuration length; 37.5 $=1,75=2,150=3$ 


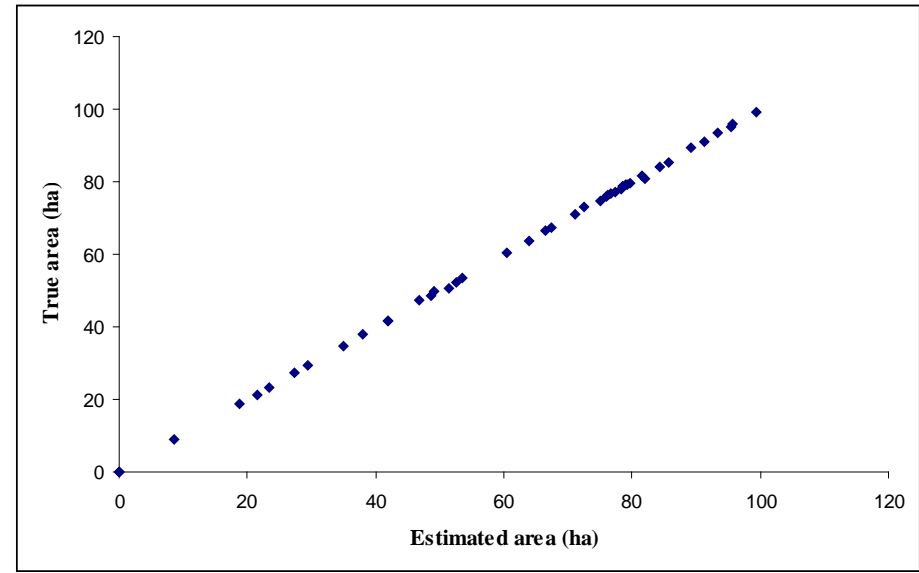

Appendix 3. An example of true and estimated values of forest class area. Level 1 classification system. For straight line configuration, sample size 16, transect length $37.5(\mathrm{~m})$, random design and transect orientation. 